\title{
Severity Estimation of Defects on Interpretation of Eddy-Current Defectograms
}

\author{
E. V. Kuzmin ${ }^{1}$, O. E. Gorbunov ${ }^{2}$, P. O. Plotnikov², V. A. Tyukin², V. A. Bashkin ${ }^{1}$ \\ DOI: $10.18255 / 1818-1015-2021-2-170-185$
}

${ }^{1}$ P. G. Demidov Yaroslavl State University, 14 Sovetskaya str., Yaroslavl 150003, Russia.

${ }^{2}$ Center of Innovative Programming, NDDLab, 144 Soyuznaya str., Yaroslavl 150008, Russia.

MSC2020: 68T09

Research article

Full text in Russian
Received January 18, 2021

After revision March 5, 2021

Accepted March 10, 2021

To ensure traffic safety of railway transport, non-destructive tests of rails are regularly carried out by using various approaches and methods, including eddy-current flaw detection methods. An automatic analysis of large data sets (defectograms) that come from the corresponding equipment is an actual problem. The analysis means a process of determining the presence of defective sections along with identifying structural elements of railway tracks in defectograms. At the same time, severity estimation of defined defects is also of great interest. This article continues the cycle of works devoted to the problem of automatic recognition of images of defects and rail structural elements in eddy-current defectograms. In the process of forming these images, only useful signals are taken into account, the threshold levels of amplitudes of which are determined automatically from eddy-current data. The article is devoted to the issue of constructing severity estimation of found defects with various lengths. The construction of the severity estimation is based on a concept of the generalized relative amplitude of useful signals. A relative amplitude is a ratio of an actual signal amplitude to a corresponding threshold level of useful signals. The generalized relative amplitude is calculated by using the entropy of the half-normal distribution, which is assumed to be a model for a probability distribution of an appearance of certain relative amplitudes in an evaluated defect. Tuning up the formula for calculating severity estimation of a defect is carried out on the basis of eddy-current records of structural elements. As a reference of the most dangerous defect, the bolted rail joint is considered. It models a fracture of a rail. A reference weak defect is a flash butt weld, a defectogram of which contains signals with low amplitude values. The proposed approach to severity estimation of defects is shown by examples.

Keywords: nondestructive testing; eddy current testing; rail flaw detection; automated analysis of defectograms; severity estimation of defects

\section{INFORMATION ABOUT THE AUTHORS}

\begin{tabular}{r|l}
$\begin{array}{r}\text { Egor V. Kuzmin } \\
\text { correspondence author }\end{array}$ & $\begin{array}{l}\text { orcid.org/0000-0003-0500-306X. E-mail: kuzmin@uniyar.ac.ru } \\
\text { Professor, Doctor of Science. }\end{array}$ \\
Oleg E. Gorbunov & $\begin{array}{l}\text { orcid.org/0000-0001-6274-9971. E-mail: gorbunovoe@nddlab.com } \\
\text { General Director, PhD. }\end{array}$ \\
Petr O. Plotnikov & $\begin{array}{l}\text { orcid.org/0000-0001-5687-7969. E-mail: plotnikovpo@nddlab.com } \\
\text { Production Engineer. }\end{array}$ \\
Vadim A. Tyukin & $\begin{array}{l}\text { orcid.org/0000-0001-9149-7435. E-mail: tyukinva@nddlab.com } \\
\text { Head of Software Development Department. }\end{array}$ \\
Vladimir A. Bashkin & $\begin{array}{l}\text { orcid.org/0000-0002-2534-1026. E-mail: bashkinva@nddlab.com } \\
\text { Professor, Doctor of Science. }\end{array}$
\end{tabular}

Funding: This work was supported by P. G. Demidov Yaroslavl State University Project № VIP-016.

For citation: E. V. Kuzmin, O. E. Gorbunov, P. O. Plotnikov, V. A. Tyukin, and V. A. Bashkin, "Severity Estimation of Defects on Interpretation of Eddy-Current Defectograms”, Modeling and analysis of information systems, vol. 28, no. 2, pp. 170-185, 2021. 


\title{
Оценка степени опасности дефектов при расшифровке вихретоковых дефектограмм
}

\author{
Е. В. Кузьмин ${ }^{1}$, О. Е. Горбунов ${ }^{2}$, П. О. Плотников ${ }^{2}$, В. А. Тюкин ${ }^{2}$, В. А. Башкин ${ }^{1}$ \\ DOI: $10.18255 / 1818-1015-2021-2-170-185$
}

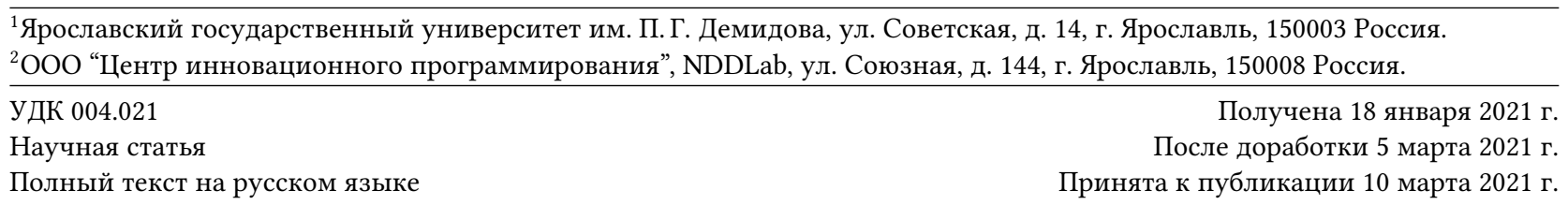

Для обеспечения безопасности движения на железнодорожном транспорте регулярно проводится неразрушающий контроль рельсов с применением различных подходов и методов, включая методы вихретоковой дефектоскопии. Актуальной задачей является автоматический анализ больших массивов данных (дефектограмм), которые поступают от соответствующего оборудования. Под анализом понимается процесс определения по дефектограммам наличия дефектных участков наряду с выявлением конструктивных элементов рельсового пути. При этом также большой интерес представляет и оценка степени опасности выявленных дефектов. Данная статья продолжает цикл работ, посвященных задаче автоматического распознавания образов дефектов и конструктивных элементов железнодорожных рельсов по вихретоковым дефектограммам. При формировании этих образов принимаются в расчет только полезные сигналы, пороговые уровни амплитуд которых определяются автоматически по вихретоковым данным. Статья посвящена задаче построения оценки степени опасности для выявленных поверхностных дефектов различной протяжённости. Построение оценки опирается на понятие обобщённой относительной амплитуды полезных сигналов. Относительная амплитуда представляет собой отношение реальной амплитуды сигнала к соответствующему пороговому уровню полезных сигналов. Обобщённая относительная амплитуда вычисляется с использованием энтропии полунормального распределения, которое предполагается модельным для распределения вероятностей появления тех или иных относительных амплитуд в оцениваемом дефекте. Настройка формулы расчёта степени опасности дефекта осуществляется на основе записей конструктивных элементов. В качестве эталонного наиболее опасного дефекта рассматривается болтовой рельсовый стык, который моделирует излом рельса. Эталонным слабым дефектом выступает электроконтактная сварка, дефектограмма которой, как правило, содержит сигналы с невысоким значением амплитуд. Предложенный подход к оценке степени опасности дефектов демонстрируется на примерах.

Ключевые слова: неразрушающий контроль рельсов; вихретоковая дефектоскопия; обнаружение дефектов; автоматический анализ дефектограмм; оценка опасности дефектов

\section{ИНФОРМАЦИЯ ОБ АВТОРАХ}

Егор Владимирович Кузьмин автор для корреспонденции

Олег Евгеньевич Горбунов

Петр Олегович Плотников

Вадим Александрович Тюкин

Владимир Анатольевич Башкин
orcid.org/0000-0003-0500-306X. E-mail: kuzmin@uniyar.ac.ru профессор, доктор физ.-мат. наук.

orcid.org/0000-0001-6274-9971. E-mail: gorbunovoe@nddlab.com генеральный директор, канд. физ.-мат. наук.

orcid.org/0000-0001-5687-7969. E-mail: plotnikovpo@nddlab.com инженер-технолог.

orcid.org/0000-0001-9149-7435. E-mail: tyukinva@nddlab.com руководитель сектора разработки.

orcid.org/0000-0002-2534-1026. E-mail: bashkinva@nddlab.com профессор, доктор физ.-мат. наук.

Финансирование: Работа выполнена в рамках инициативной НИР ЯрГУ им. П. Г. Демидова № VIP-016.

Для цитирования: E. V. Kuzmin, O. E. Gorbunov, P. O. Plotnikov, V. A. Tyukin, and V. A. Bashkin, "Severity Estimation of Defects on Interpretation of Eddy-Current Defectograms”, Modeling and analysis of information systems, vol. 28, no. 2, pp. 170-185, 2021.

(c) Кузьмин Е. В., Горбунов О. Е., Плотников П. О., Тюкин В. А., Башкин В. А., 2021

Эта статья открытого доступа под лицензией СС BY license (https://creativecommons.org/licenses/by/4.0/). 


\section{Введение}

Для обеспечения безопасности движения на железнодорожном транспорте регулярно проводится неразрушающий контроль рельсов с применением различных подходов и методов, включая методы вихретоковой дефектоскопии. Актуальной задачей является автоматический анализ [1-3] больших массивов данных (дефектограмм), которые поступают от соответствующего оборудования. Под анализом понимается процесс определения по дефектограммам наличия дефектных участков наряду с выявлением конструктивных элементов рельсового пути. При этом также большой интерес представляет оценка степени опасности выявленных дефектов.

Данная статья продолжает цикл работ [4-8], посвященных задаче автоматического распознавания образов дефектов и конструктивных элементов железнодорожных рельсов по дефектограммам многоканальных вихретоковых дефектоскопов. Дефектограммы разбиваются на фрагменты (блоки анализа), каждый из которых обрабатывается отдельно. В текущем блоке анализа с использованием алгоритма из статей [4-6] для каналов данных происходит выделение полезных сигналов, которые группируются в отметки. Найденные отметки подлежат дальнейшей классификации с применением нейронных сетей. В статье [7] решалась задача распознавания записей небольших конструктивных элементов (длиной до 157 мм) следующих трёх типов: 1) болтовой стык с прямым или скошенным соединением рельсов, 2) электроконтактная сварка рельсов и 3) алюминотермитная сварка рельсов. В статье [8] проводилось распознавание записей длинных (от 420 мм до 3220 мм) конструктивных элементов рельсового пути двух классов: 1) счётчик осей подвижного состава, 2) пересечение рельсовых путей. Отметки, которые не были отнесены к тому или иному типу конструктивных элементов, классифицируются как условные дефекты.

Эта статья посвящена задаче построения оценки степени опасности для выявленных поверхностных дефектов различной протяжённости. Построение оценки опирается на понятие обобщённой относительной амплитуды полезных сигналов. Относительная амплитуда представляет собой отношение реальной амплитуды сигнала к соответствующему пороговому уровню полезных сигналов. Обобщённая относительная амплитуда вычисляется с использованием энтропии полунормального распределения, которое предполагается модельным для распределения вероятностей появления тех или иных относительных амплитуд в оцениваемой отметке.

Настройка формулы расчёта степени опасности отметки осуществляется на основе записей конструктивных элементов. В качестве эталонного наиболее опасного дефекта рассматривается болтовой рельсовый стык, который моделирует излом рельса. Эталонным слабым дефектом выступает электроконтактная сварка, дефектограмма которой, как правило, содержит сигналы с невысоким значением амплитуд.

В статье рассматривается обобщённое устройство в виде 12-разрядного вихретокового дефектоскопа с 15 каналами данных (на один рельс). Каналы данных соответствуют физическим датчикам, которые последовательно располагаются на поверхности рельса перпендикулярно направлению движения дефектоскопа. Значения амплитуд сигналов каждого канала регистрируются дефектоскопом виде целых чисел от -2048 до 2047. Шаг сканирования дефектоскопа - 1 мм.

Интерес представляют амплитуды только полезных сигналов. Отметим, что применяемый ранее алгоритм определения порогового уровня амплитуд полезных сигналов [5, 6], рассчитанный на фрагменты вихретоковых дефектограмм, подавляющее большинство сигналов в которых составляет рельсовый шум, был скорректирован в работе [4]. Были учтены ситуации с возможным наличием на анализируемом фрагменте вихретоковой дефектограммы большого количество полезных сигналов (например, от протяженных поверхностных дефектов, длина которых может составлять несколько сотен метров).

Далее в статье предполагается, что полезные сигналы определяются именно по соответствующим скорректированным пороговым уровням. 


\section{1. Предварительные сведения}

Поскольку далее в статье предполагается, что вероятности относительных амплитуд сигналов произвольной отметки имеют полунормальное распределение, а ключевым моментом при расчёте степени опасности отметки является применение энтропии, дадим сначала необходимые предварительные сведения, касающиеся используемых понятий.

Пусть непрерывная случайная величина $X$ имеет полунормальное распределение вероятностей (half-normal distribution). Тогда функция плотности распределения вероятностей имеет вид

$$
f(x)=\frac{\sqrt{2}}{\sigma \sqrt{\pi}} e^{-\frac{x^{2}}{2 \sigma^{2}}}, x \geqslant 0
$$

где $\sigma$ - среднеквадратическое отклонение случайной величины относительно нуля.

Для полунормального распределения имеем

$$
\begin{aligned}
\int_{0}^{+\infty} f(x) d x & =\int_{0}^{+\infty} \frac{\sqrt{2}}{\sigma \sqrt{\pi}} e^{-\frac{x^{2}}{2 \sigma^{2}}} d x=1, \\
\int_{0}^{+\infty} x^{2} f(x) d x & =\int_{0}^{+\infty} x^{2} \frac{\sqrt{2}}{\sigma \sqrt{\pi}} e^{-\frac{x^{2}}{2 \sigma^{2}}} d x=\sigma^{2} .
\end{aligned}
$$

Рассмотрим формулу энтропии полунормального распределения вероятностей, записанную с использованием натурального логарифма:

$$
\begin{aligned}
H[X] & =-\int_{0}^{+\infty} f(x) \ln f(x) d x=-\int_{0}^{+\infty} f(x)\left(\ln \frac{\sqrt{2}}{\sigma \sqrt{\pi}}-\frac{x^{2}}{2 \sigma^{2}} \ln e\right) d x= \\
& =\ln \frac{\sigma \sqrt{\pi}}{\sqrt{2}} \int_{0}^{+\infty} f(x) d x+\frac{1}{2 \sigma^{2}} \int_{0}^{+\infty} x^{2} f(x) d x=\ln \frac{\sigma \sqrt{\pi}}{\sqrt{2}}+\frac{1}{2}= \\
& =\ln \sigma+\ln \frac{\sqrt{\pi}}{\sqrt{2}}+\frac{1}{2} \approx \ln \sigma+0,726 .
\end{aligned}
$$

Отсюда получаем следующую формулу вычисления $\sigma$ через энтропию

$$
\sigma \approx e^{H[X]-0,726} .
$$

\section{2. Расчет степени опасности дефекта}

Шаг 0. Нормирование амплитуд полезных сигналов. С помощью следующей функции, написанной на языке Python 3, происходит подготовка исходных данных для расчёта степени опасности найденной отметки. Значение амплитуды каждого сигнала отметки делится на соответствующий пороговый уровень полезных сигналов и округляется с точностью до $1 /$ coef, где соеf = 10 . Из полученного результата вычитается единица, т. е. осуществляется смещение на 1. Затем подсчитывается частота каждого различного значения таких относительных амплитуд со смещением.

В этой функции ЕС[0:49999,0:14] - это матрица анализируемых исходных вихретоковых данных, т. е. фрагмент дефектограммы длиной 50 метров с данными от 15 каналов, Threshold[0:14] список положительных и отрицательных пороговых значений амплитуд полезных сигналов для 15 каналов, start и end - координаты начала и конца текущей отметки. Результатом работы функции является массив $R$ частот амплитуд полезных сигналов отметки, нормированных относительно соответствующего порогового уровня сигналов с последующим вычитанием единицы, умножением 
на coef и округлением до ближайшего целого. Другими словами, элемент $R[i]$ представляет собой количество найденных значений $i=\operatorname{round}(\operatorname{coef} \cdot k)$, где $k-$ это отношение полезного сигнала отметки к соответствующему пороговому уровню сигналов без единицы, a round - функция округления до ближайшего целого числа. С учётом специфики хранения данных в массиве $R$ далее будем называть $i$ просто значением смещённой относительной амплитуды полезного сигнала, а $R[i]-$ частотой появления в отметке смещённой относительной амплитуды со значением $i$.

Число 10, выбранное в качестве значения для константы соеf, означает, что смещённые относительные амплитуды различаются с точностью до одной десятой.

def Data_Preparation(Threshold, EC, start, end, coef, R):

for $j$ in range $(0,15)$ :

thplus $=$ Threshold $[j]$. thplus

thmins $=$ Threshold $[j] \cdot$ thmins

for $i$ in range (start, end +1$)$ :

$\mathrm{ec}=\mathrm{EC}[i, j]$

if ec > thplus:

$\mathrm{R}[\operatorname{int}($ coef $*((\mathrm{ec} /$ thplus $)-1)+0.5)]+=1$

if ec < thmins:

$\mathrm{R}[\operatorname{int}(\operatorname{coef} *((\mathrm{ec} / \mathrm{thmins})-1)+0.5)]+=1$

Шаг 1. Вычисление энтропии. Рассмотрим массив $R[0: n]$ частотного распределения значений смещённых относительных амплитуд $X=\{0,1, \ldots, n\}$ всех полезных сигналов текущей отметки. Число $n$ - значение максимальной смещённой относительной амплитуды этой отметки. Произведём вычисление энтропии $H$ для множества $X=\{0,1, \ldots, n\}$ по формуле

$$
H=-\sum_{i=0}^{n} p_{i} \ln p_{i}, \text { где } \sum_{i=0}^{n} p_{i}=1,
$$

$p_{i}$ - вероятность появления в отметке смещённой относительной амплитуды со значением $i \in X$.

Следующая функция на языке Python 3 реализует эту формулу. Кроме набора данных $R$ и значения $n$ на вход функции подаётся суммарное значение $c t r$ всех элементов массива $R$. В качестве результата функция выдаёт значение $H$ энтропии для набора данных $X$.

def Get_Entropy(R, n, ctr):

$\mathrm{H}=0$

for $i$ in range $(0, n+1)$ :

if $\mathrm{R}[\mathrm{i}]>0$ :

$\mathrm{H}+=(\mathrm{R}[\mathrm{i}] / \mathrm{ctr}) * \operatorname{math} \cdot \log (\operatorname{ctr} / \mathrm{R}[\mathrm{i}])$

return $\mathrm{H}$

Шаг 2. Среднеквадратическое отклонение. Допуская, что вероятность появления в отметке той или иной смещённой относительной амплитуды в приближении имеет полунормальное распределение, вычислим для значений этих относительных амплитуд их среднеквадратическое отклонение от нуля через полученную энтропию $H$ по следующей формуле:

$$
\sigma=\frac{e^{H-0,726}}{\operatorname{coef}} .
$$

Деление на коэффициент соеf проводится с учётом особенности формирования массива $R[0: n]$, с помощью которого осуществляется хранение частот значений смещённых относительных амплитуд полезных сигналов. 
Шаг 3. Правило трёх сигм и децибель. Воспользуемся правилом трёх сигм, чтобы построить обобщённый уровень относительных амплитуд полезных сигналов и переведём его в децибелы:

$$
\text { Amplitude }=20 \lg (1+3 \sigma),
$$

где Amplitude - обобщённый уровень относительных амплитуд в децибелах.

Шаг 4. Оценка степени опасности. На основе обобщённого уровня Amplitude относительных амплитуд, выраженного в децибелах, производится расчёт степени опасности Severity для текущей отметки. При этом принимаются во внимание следующие параметры: Scale - градация (количество пунктов) степени опасности, minAmpl и maxAmpl - наименьшее и наибольшее пороговые значения обобщённого уровня относительных амплитуд. Параметр $\min A m p l$ позволяет игнорировать незначительные отметки, для которых не предусмотрена даже минимальная степень опасности. Значение maxAmpl устанавливает порог, при превышении которого более не имеет смысла различать максимально опасные отметки. В этом случае всем им присваивается предельный уровень опасности. Наивысший уровень опасности соответствует значению Severity = 1. С увеличением значения Severity уменьшается и степень опасности отметки.

Приведённая ниже функция на языке Python 3 реализует последний этап вычисления оценки степени опасности отметки.

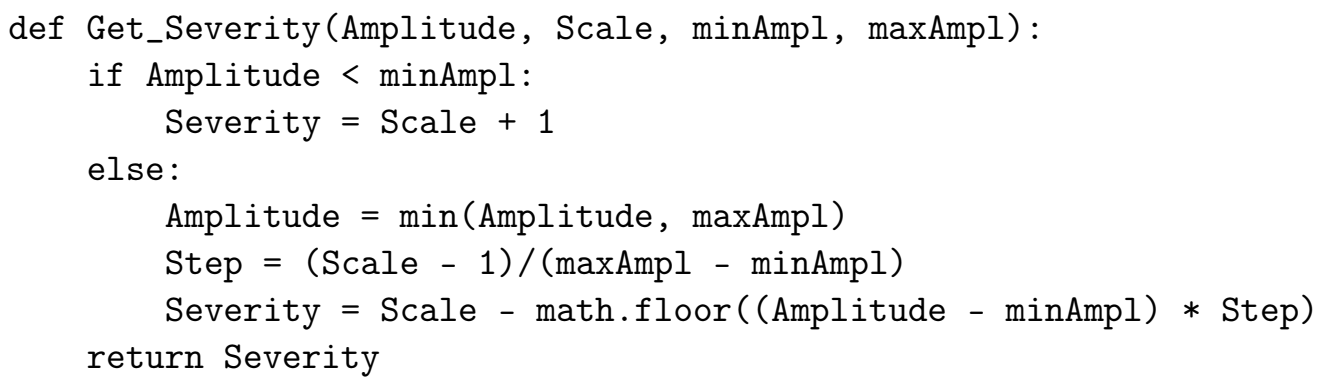

Здесь math.floor() - функция округления числа до ближайшего наименьшего целого. Отметим, что при дальнейшем анализе все отметки, получившие значение Severity paвное Scale + 1, считаются незначительными по степени опасности и удаляются из списка дефектов.

\section{3. Обоснование этапов расчёта}

Одним из ключевых моментов при расчёте степени опасности отметки является введение понятия обобщённой относительной амплитуды. При некотором «усреднении» обобщённая относительная амплитуда позволяет получить наглядное представление о том, во сколько раз амплитуды полезных сигналов отметки больше соответствующих найденных пороговых уровней (полезных сигналов). На практике рост значения обобщённой амплитуды регистрируемых сигналов напрямую связан со степенью изношенности поверхности катания тестируемого фрагмента рельса.

В качестве «усредняющей процедуры» применяется правило трёх сигм при модельном допущении, что вероятности смещённых относительных амплитуд отметки соответствуют полунормальному закону распределения. По этому правилу предполагается, что для текущей отметки почти все относительные амплитуды без смещения на единицу ограничены сверху значением $1+3 \cdot \sigma$, где $\sigma$ - среднеквадратическое отклонение от нуля, вычисленное через энтропию, для значений смещённых относительных амплитуд полезных сигналов.

Важно отметить, что именно использование энтропии при вычислении среднеквадратического отклонения $\sigma$ позволяет получать стабильный ожидаемый результат при построении оценки степени опасности поверхностного дефекта. Вычисление среднеквадратического отклонения напрямую 

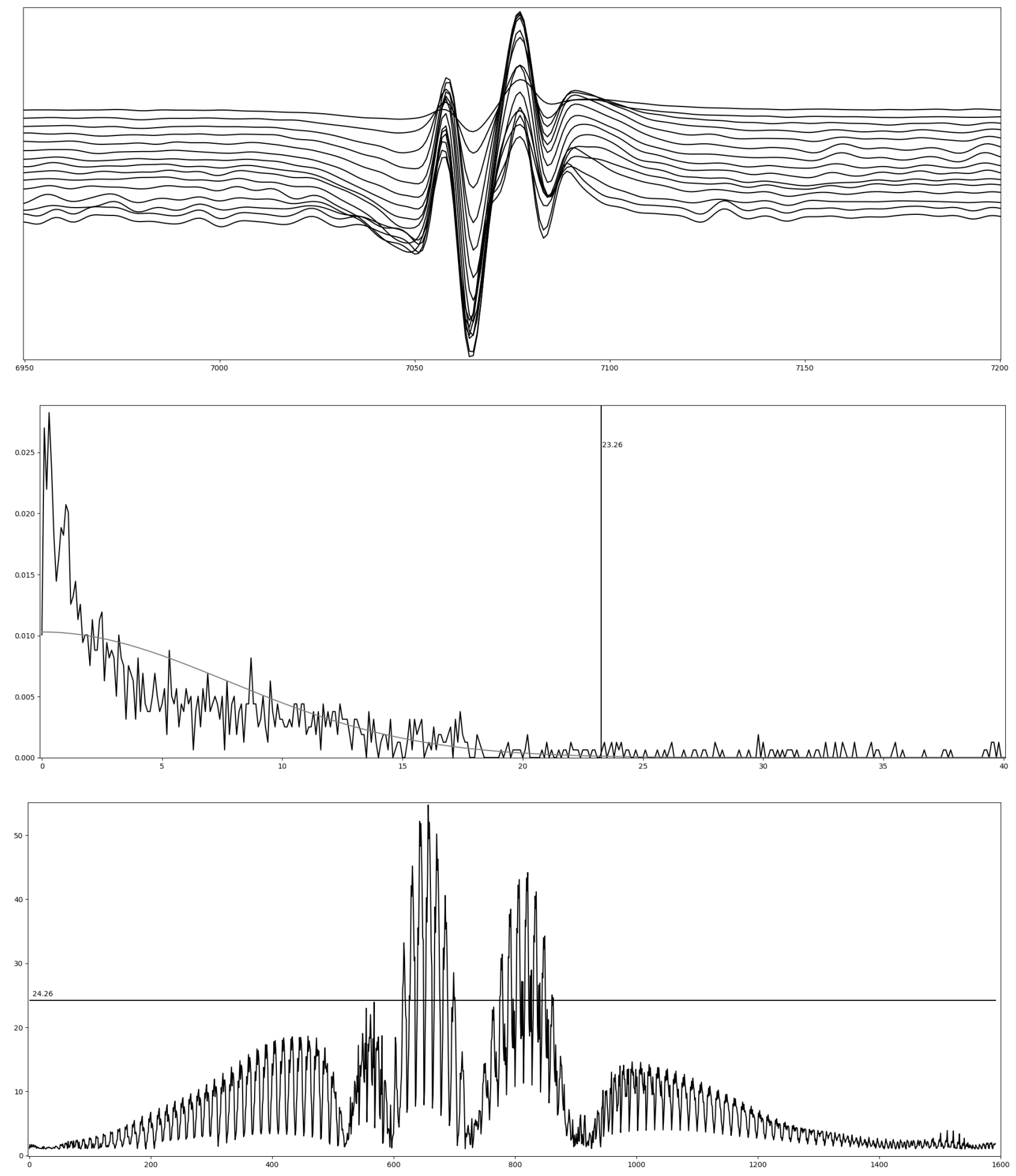

Fig. 1. Straight bolted joint (at the top), $250 \mathrm{~mm}$, Severity $=1$. Probability distributions of shifted relative amplitudes (at the centre). Values of relative amplitudes (at the bottom). Useful signals are $45,49 \%$
Pис. 1. Прямой болтовой стык (вверху), 250 мм, Severity $=1$. Распределения вероятностей смещённых относительных амплитуд (по центру). Значения относительных амплитуд (внизу). Полезных сигналов 45,49\% 

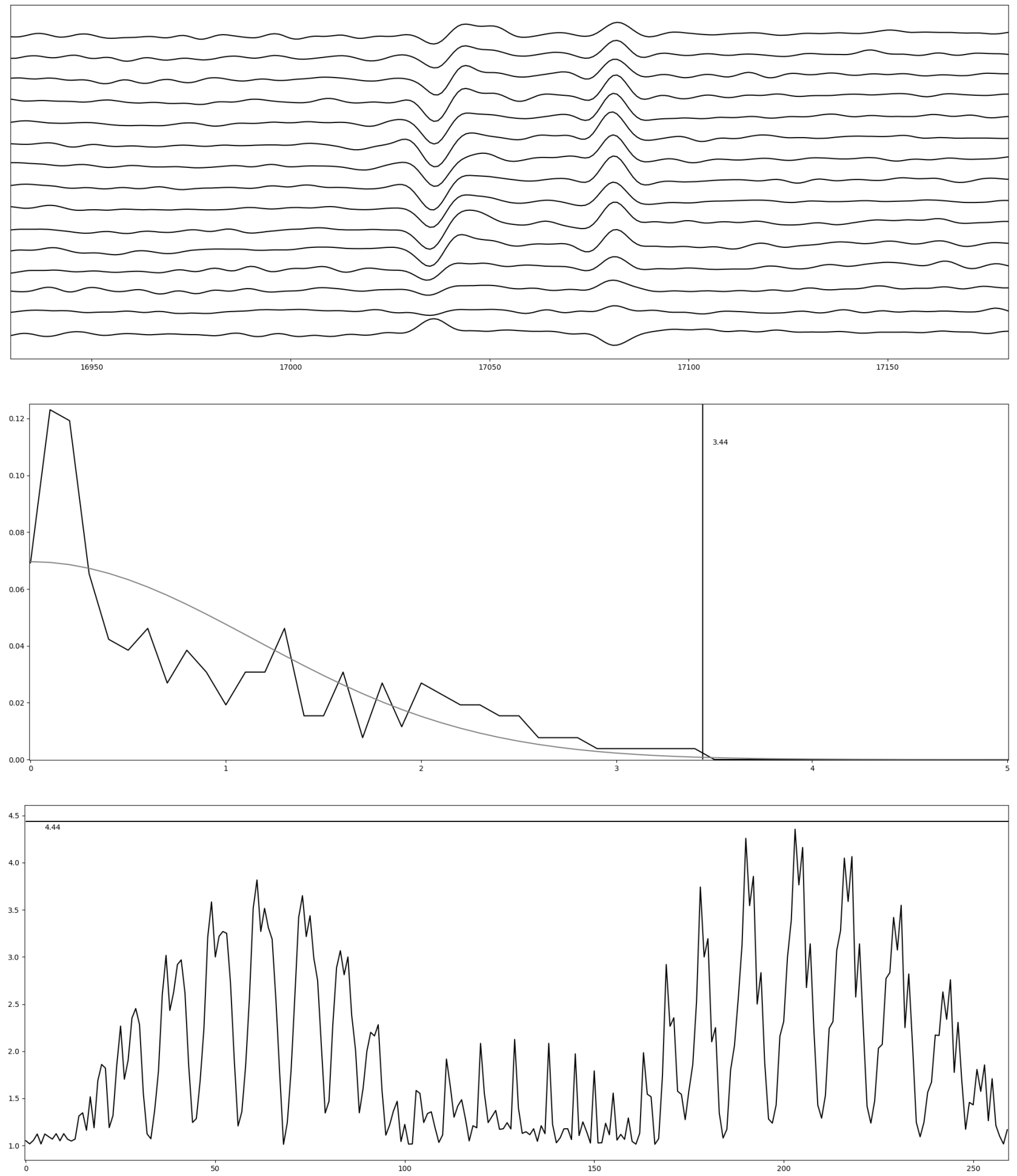

Fig. 2. Flash butt weld (at the top), $250 \mathrm{~mm}$, Severity $=9$. Probability distributions of shifted relative amplitudes (at the centre). Values of relative amplitudes (at the bottom). Useful signals are $7,43 \%$
Рис. 2. Электроконтактная сварка (вверху), 250 мм, Severity = 9. Распределения вероятностей смещённых относительных амплитуд (по центру). Значения относительных амплитуд (внизу). Полезных сигналов 7,43\% 

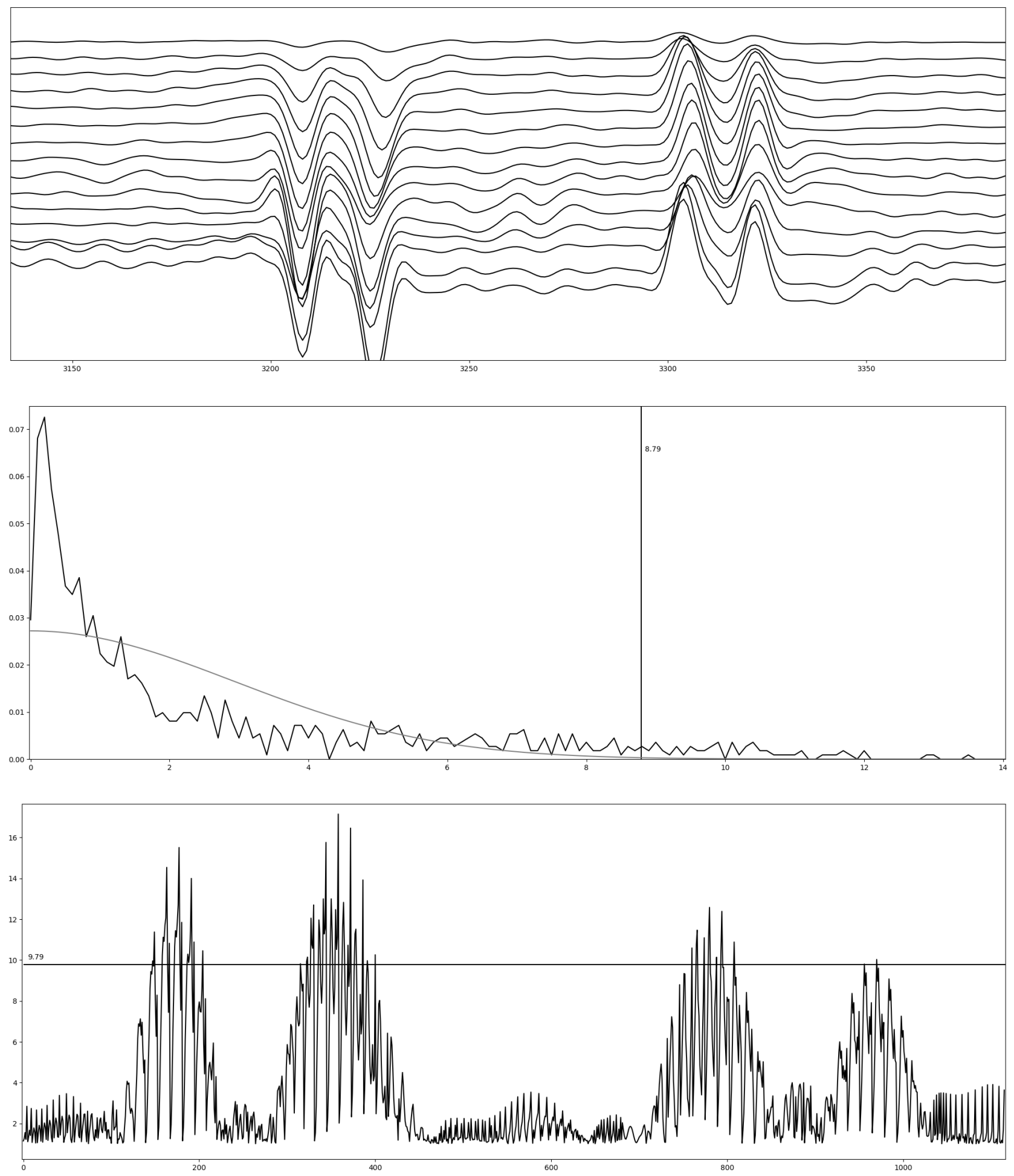

Fig. 3. Aluminothermic rail weld (at the top), $250 \mathrm{~mm}$, Severity $=5$. Probability distributions of shifted relative amplitudes (at the centre). Values of relative amplitudes (at the bottom). Useful signals are $31,89 \%$
Рис. 3. Алюминотермитная сварка (вверху), 250 мм, Severity $=5$. Распределения вероятностей смещённых относительных амплитуд (по центру). Значения относительных амплитуд (внизу). Полезных сигналов 31,89\% 

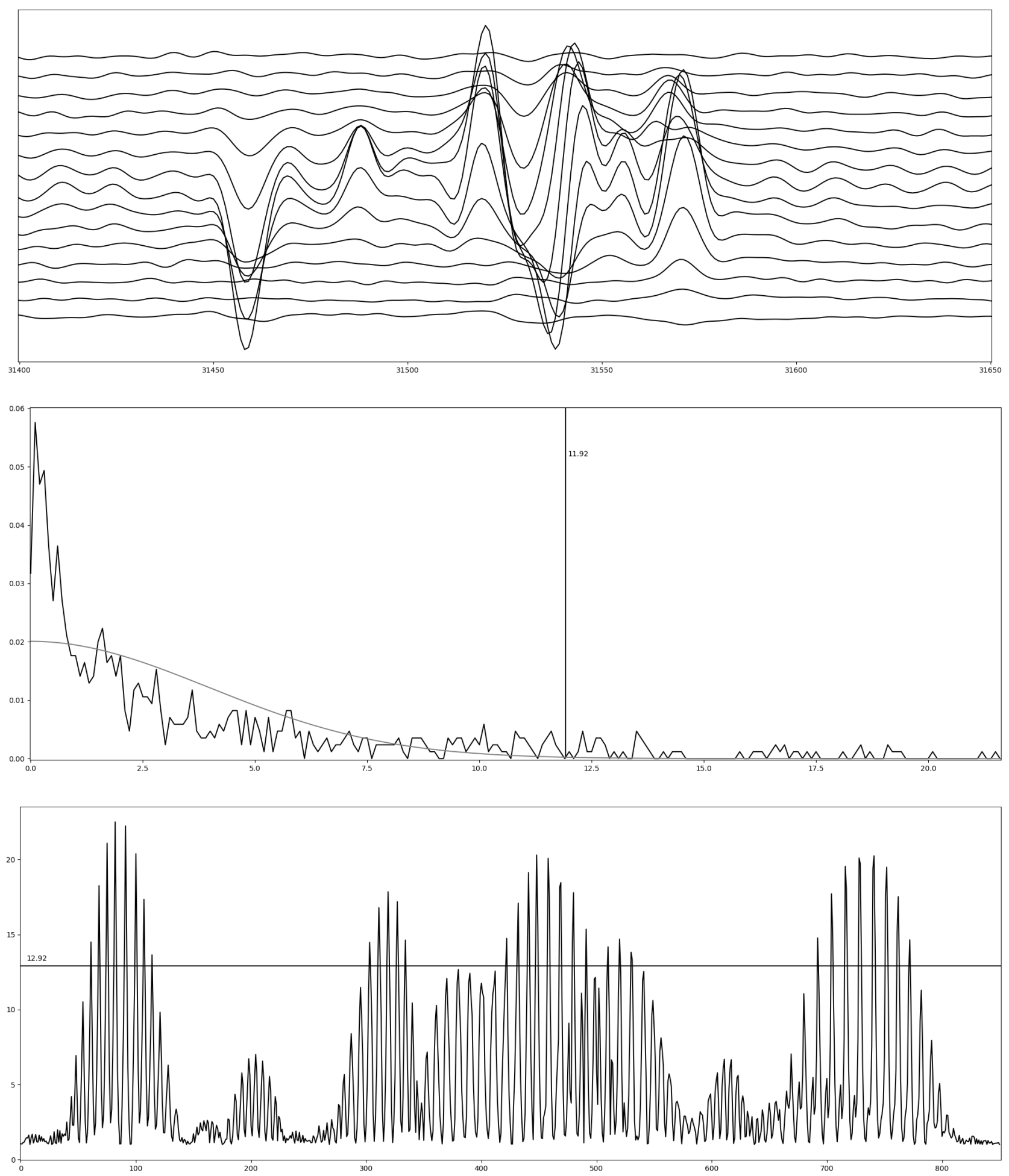

Fig. 4. Single surface defect (at the top), $250 \mathrm{~mm}$, Severity $=4$. Probability distributions of shifted relative amplitudes (at the centre). Values of relative amplitudes (at the bottom). Useful signals are $24,31 \%$
Рис. 4. Одиночный поверхностный дефект (вверху), 250 мм, Severity = 4. Распределения вероятностей смещённых относительных амплитуд (по центру). Значения относительных амплитуд (внизу). Полезных сигналов $24,31 \%$ 

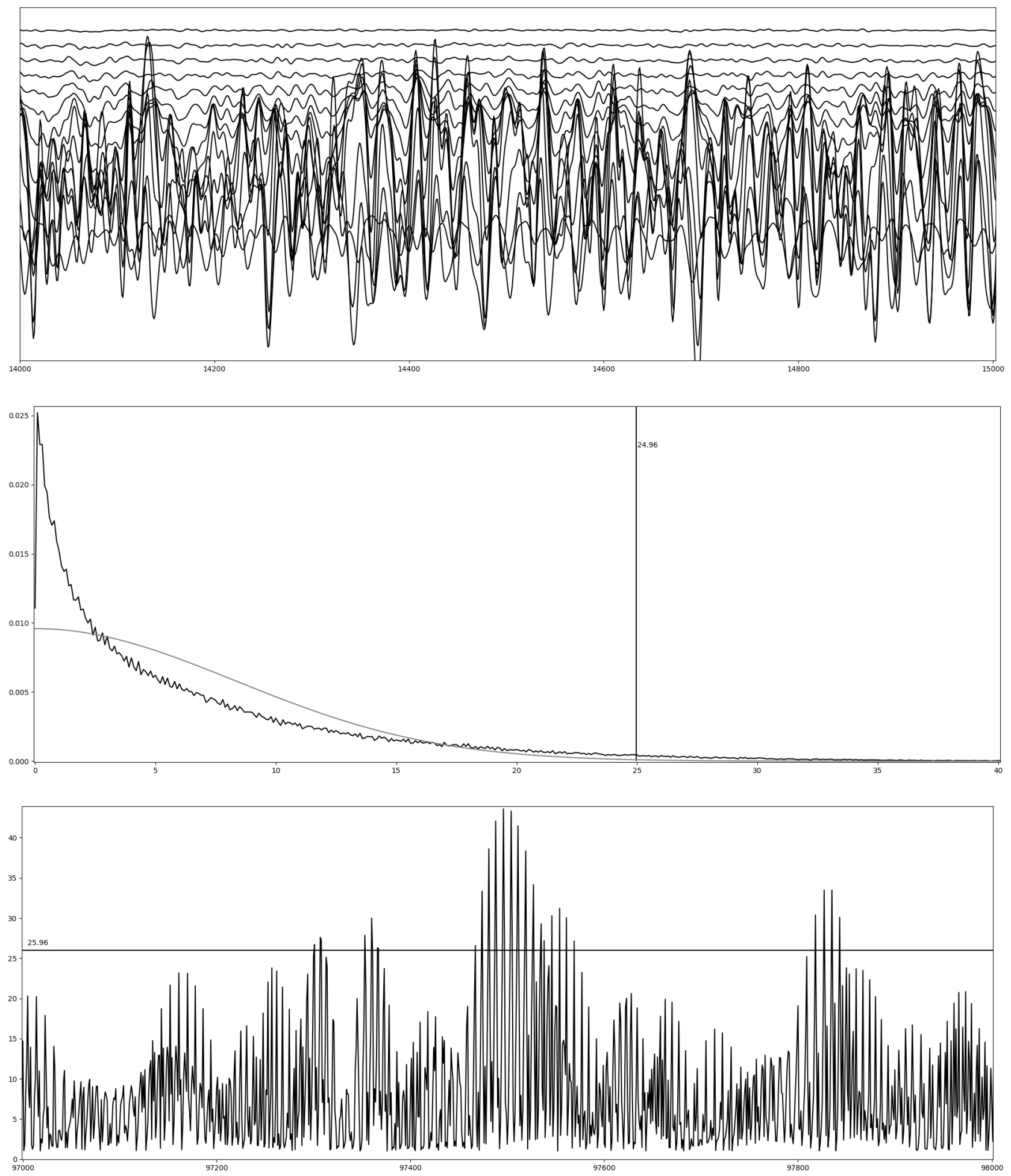

Fig. 5. One-meter fragment of 50-meter gauge side defect (at the top), Severity $=1$. The first thousand values of relative amplitudes of the fragment signals (at the bottom). Useful signals are $49,74 \%$
Рис. 5. Метровый фрагмент 50-метрового дефекта рабочей грани (вверху), Severity = 1 . Первая тысяча значений относительных амплитуд сигналов фрагмента (внизу). Полезных сигналов 49,74\% 

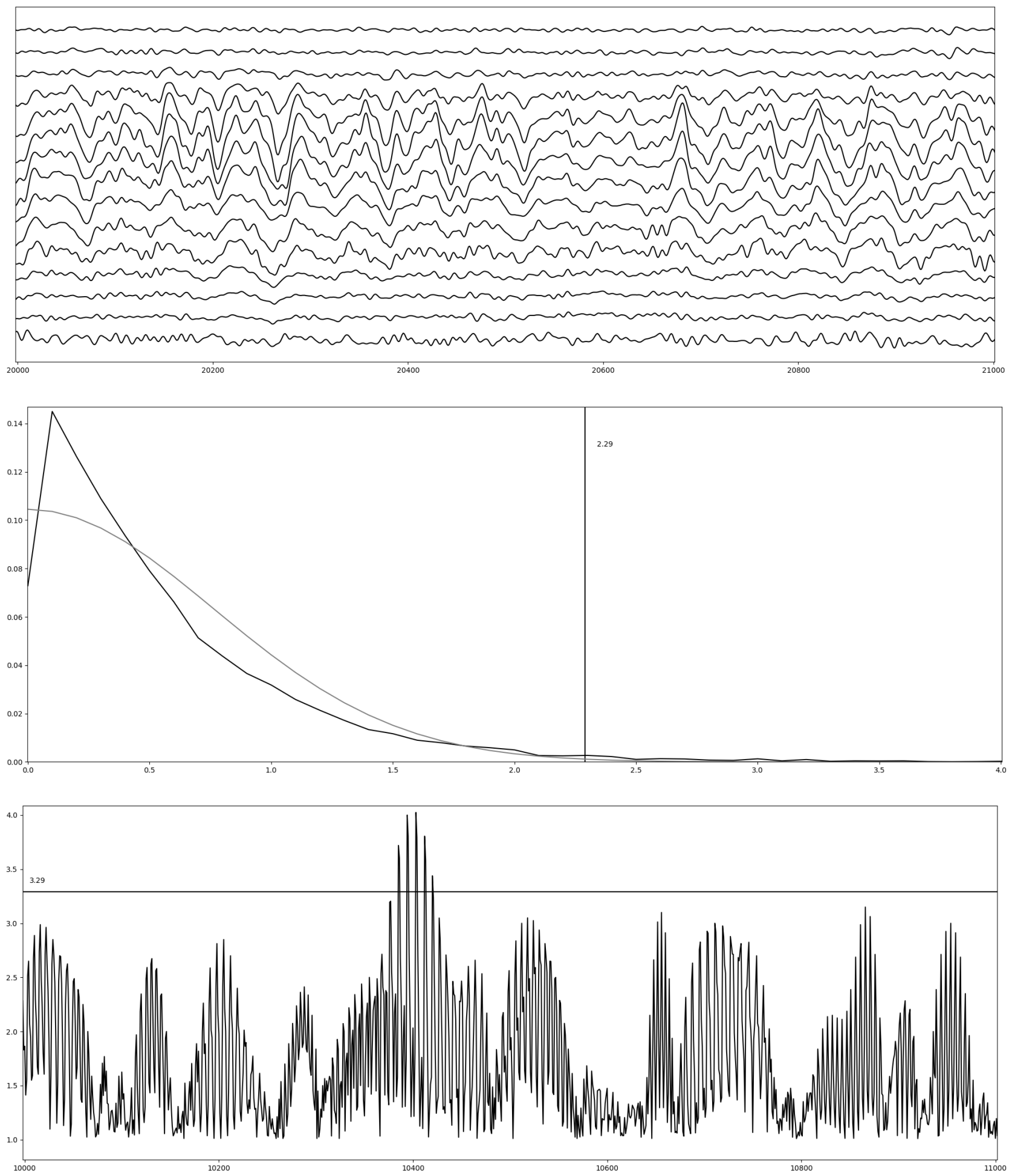

Fig. 6. One-meter fragment of 9-meter surface defect (at the top), Severity $=10$. The first thousand values of relative amplitudes of the fragment signals (at the bottom). Useful signals are 13,59\%
Рис. 6. Метровый фрагмент 9-метрового дефекта поверхности катания (вверху), Severity $=10$. Первая тысяча значений относительных амплитуд сигналов фрагмента (внизу). Полезных сигналов 13,59\% 

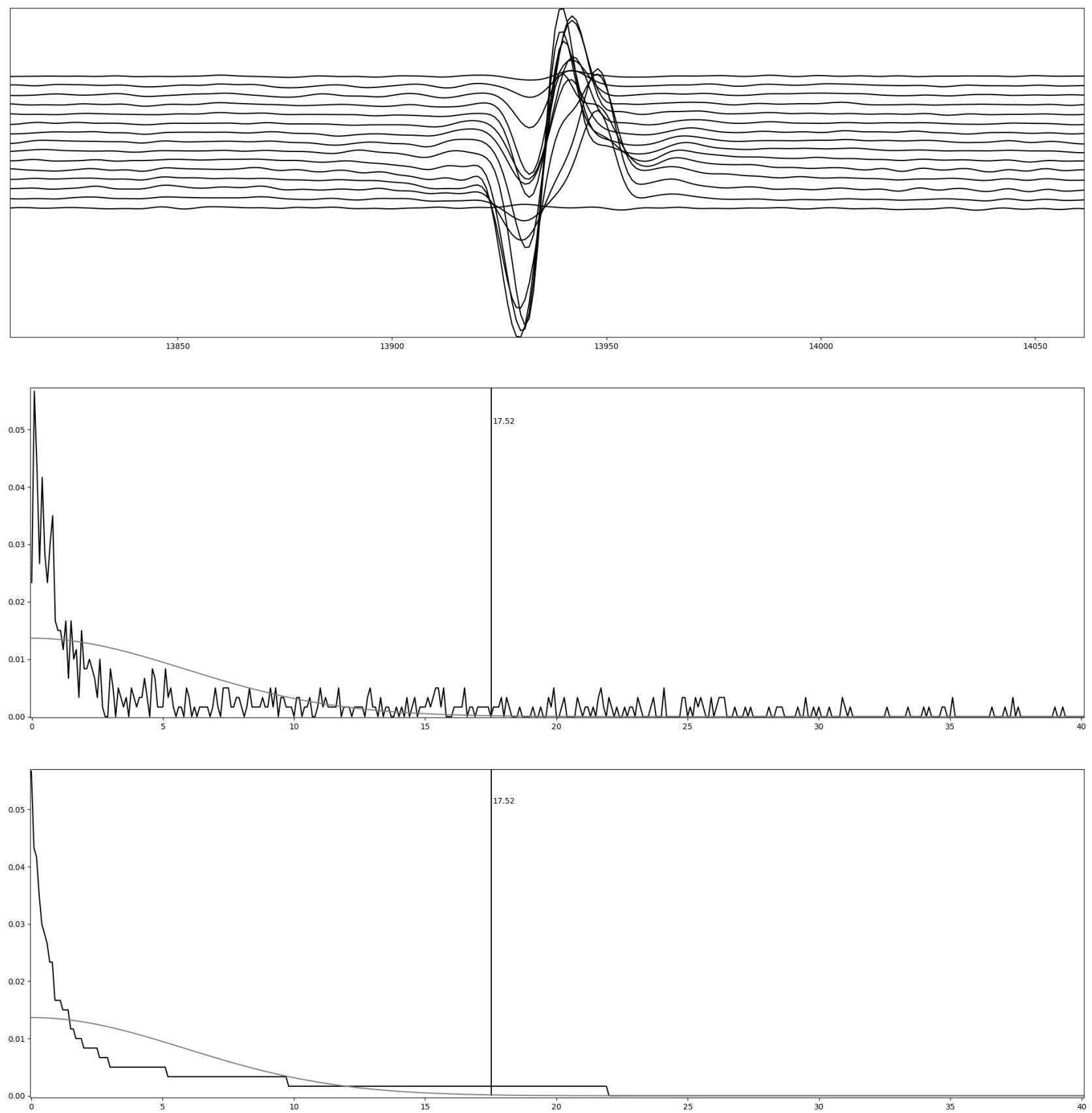

Fig. 7. Single short surface defect (on the top), $250 \mathrm{~mm}$, Severity $=2$. Real distribution (at the centre) and imaginary distribution (at the bottom) of probabilities of shifted relative amplitudes of useful signals. Useful signals are $17,14 \%$
Рис. 7. Одиночный короткий поверхностный дефект (вверху), 250 мм, Severity $=2$. Реальное распределение (по центру) и мнимое распределение (внизу) вероятностей смещённых относительных амплитуд полезных сигналов. Полезных сигналов 17,14\% 

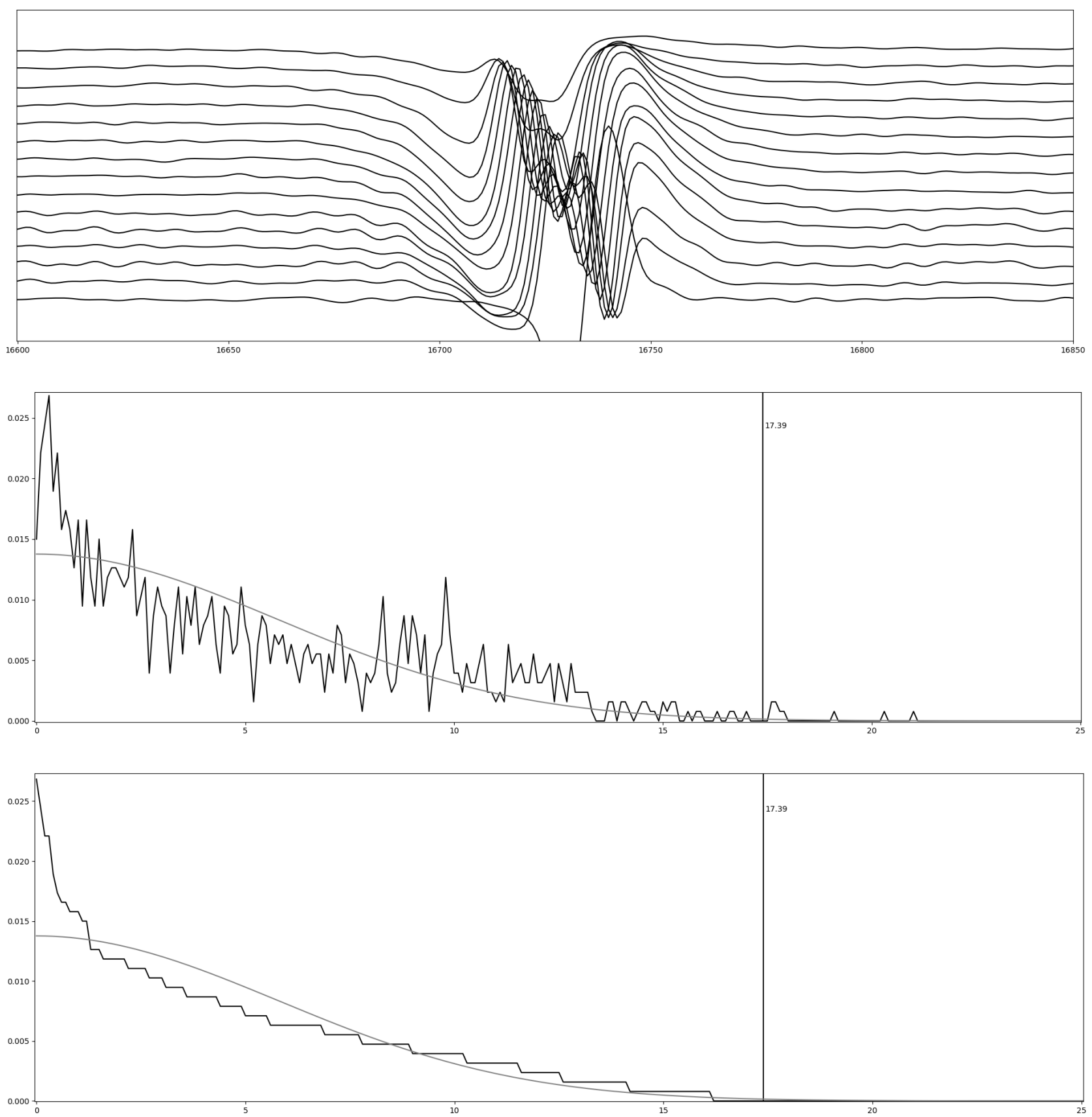

Fig. 8. Beveled bolted joint, $250 \mathrm{~mm}$, Severity $=2$. Real distribution (at the centre) and imaginary distribution (at the bottom) of probabilities of shifted relative amplitudes of useful signals. Useful signals are $36,20 \%$
Рис. 8. Косой болтовой стык (вверху), 250 мм, Severity $=2$. Реальное распределение (по центру) и мнимое распределение (внизу) вероятностей смещённых относительных амплитуд полезных сигналов. Полезных сигналов 36,20\% 
или же с применением алгоритма из статей [4-6] нецелесообразно, так как совершенно не гарантируется, что полученные значения будут согласовываться с поставленными целями по причине частого завышения или занижения (относительно ожиданий) вычисляемого значения $\sigma$.

На рис. 1-6 для различных фрагментов дефектограмм (вверху) показаны (по центру) реальное и модельное распределения вероятностей смещённых относительных амплитуд полезных сигналов с вертикальной линией отсечки для значения $3 \cdot \sigma$. Более того, в нижней части рисунков приведены выстроенные в линию значения относительных амплитуд полезных сигналов с горизонтальной линией отсечки для обобщённой относительной амплитуды, равной $1+3 \cdot \sigma$.

Отметим, что последующий перевод обобщённой относительной амплитуды в децибелы это стандартное действие при работе с подобными величинами. Различное восприятие человеком близких слабых и близких сильных амплитуд приводит к необходимости промежуточного использования логарифмической шкалы для того, чтобы далее иметь возможность адекватного перехода к «линейным» уровням/степеням опасности.

Следующим ключевым моментом при построении оценки степени опасности является настройка её шкалы.

Для настройки шкалы степеней опасности предлагается использовать записи конструктивных элементов, которые регулярно встречаются на дефектограммах. В качестве эталонной отметки, имеющей максимальный уровень опасности Severity $=1$, удобно рассматривать запись болтового рельсового стыка (см. рис. 1). В данной ситуации болтовой рельсовый стык будет играть роль излома, т. е. роль самого опасного дефекта. В качестве эталонного слабого дефекта, который имеет смысл начинать отслеживать, выступает электроконтактная сварка (см. рис. 2). Как правило, образ электроконтактной сварки формируется сигналами с относительно невысоким значением амплитуды. При таком подходе алюминотермитная сварка будет давать отметку с промежуточным уровнем опасности (см. рис. 3).

На данный момент времени настройка формулы оценки степени опасности отметки осуществлена с применением следующих значений параметров: Scale $=10, \operatorname{minAmpl}=9$ и $\operatorname{maxAmpl}=27$. Оценка Severity для отметок, представленных на всех рисунках статьи, произведена с использованием именно этих значений параметров $\operatorname{minAmpl}$ и $\operatorname{maxAmpl}$ по десятибалльной шкале.

Наконец, обратим внимание на ещё один параметр соеf $=10$, использующийся при построении массива частот смещённых относительных амплитуд $R[0: n]$. Этот параметр позволяет автоматически занижать степень опасности для тех дефектов, которые на записи имеют совсем не большое количество полезных сигналов. Несмотря на то что амплитуды сигналов этих дефектов могут принимать «зашкаливающие» значения, факт их небольшого количества означает, что соответствующие реальные поверхностные дефекты обладают скромными размерами и не являются настолько опасными, как может показать обобщённая относительная амплитуда.

Однако для таких отметок из-за небольшого количества полезных сигналов массив $R[0: n]$ получается разреженным (содержит нулевые внутренние элементы). При вычислении обобщённой относительной амплитуды через энтропию наблюдается эффект сжатия массива $R[0: n]$, поскольку он воспринимается плотным (без нулевых элементов в середине массива) и упорядоченным по убыванию. Это приводит к необходимому занижению обобщённой относительной амплитуды, по которой далее строится оценка степени опасности отметки.

Описанный эффект сжатия и сортировки набора данных $R[0: n]$ можно наблюдать на рис. 7. Для сравнения на рис. 8 приводится похожий случай, но с достаточно плотным распределением частот амплитуд сигналов, для которого эффект сжатия почти не заметен. На этих рисунках по центру представлен реальный массив распределения частот смещённых относительных амплитуд, а в нижней части рисунков показано то, как этот массив воспринимается при вычислении энтропии (для полунормального распределения). Здесь значения элементов массива $R$ отображаются 
в нормированном виде относительно общей суммы значений всех элементов массива $R$, т. е. по сути приведены графики плотности распределения вероятностей значений смещённых относительных амплитуд полезных сигналов.

\section{Заключение}

Предложенный в статье подход к оценке степени опасности поверхностных дефектов различной протяжённости хорошо показал себя на практике при неразрушающем контроле рельсов. Алгоритм оценки успешно применяется в рамках аппаратно-программного комплекса вихретоковой дефектоскопии при оценке степени опасности дефектов поверхности катания рельсов.

\section{References}

[1] A. A. Markov and E. A. Kuznetsova, Rails flaw detection. Formation and analysis of signals. Book 1. Principles. St. Petersburg: KultInformPress, 2010.

[2] A. A. Markov and E. A. Kuznetsova, Rails flaw detection. Formation and analysis of signals. Book 2. Data interpretation. St. Petersburg: Ultra Print, 2014.

[3] V.F. Tarabrin, A. V. Zverev, O. E. Gorbunov, and E. V. Kuzmin, "About Data Filtration of the Defectogram Automatic Interpretation by Hardware and Software Complex ASTRA”, NDT World, vol. 64, no. 2, pp. 5-9, 2014.

[4] E. V. Kuzmin, O. E. Gorbunov, P. O. Plotnikov, V. A. Tyukin, and V. A. Bashkin, "An Algorithm for Correcting Levels of Useful Signals on Interpretation of Eddy-Current Defectograms", Modeling and Analysis of Information Systems, vol. 28, no. 1, pp. 74-88, 2021.

[5] E. V. Kuzmin, O. E. Gorbunov, P. O. Plotnikov, and V. A. Tyukin, "Finding the Level of Useful Signals on Interpretation of Magnetic and Eddy-Current Defectograms", Automatic Control and Computer Sciences, vol. 52, no. 7, pp. 658-666, 2018.

[6] E. V. Kuzmin, O. E. Gorbunov, P. O. Plotnikov, and V. A. Tyukin, "An Efficient Algorithm for Finding the Level of Useful Signals on Interpretation of Magnetic and Eddy Current Defectograms”, Automatic Control and Computer Sciences, vol. 52, no. 7, pp. 867-870, 2018.

[7] E. V. Kuzmin, O. E. Gorbunov, P. O. Plotnikov, V. A. Tyukin, and V. A. Bashkin, "Application of Neural Networks for Recognizing Rail Structural Elements in Magnetic and Eddy Current Defectograms", Automatic Control and Computer Sciences, vol. 53, no. 7, pp. 628-637, 2019.

[8] E. V. Kuzmin, O. E. Gorbunov, P. O. Plotnikov, V. A. Tyukin, and V. A. Bashkin, "Application of Convolutional Neural Networks for Recognizing Long Structural Elements of Rails in Eddy-Current Defectograms”, Modeling and Analysis of Information Systems, vol. 27, no. 3, pp. 316-329, 2020. 